\title{
Optimization Methods used for Automatic Image Annotation/Retrieval: A Survey
}

\author{
Ashitha Jose \\ Department of CSE \\ College of Engineering Poonjar \\ Kottyam, Kerala - India
}

\author{
Sreekumar K. \\ Department of CSE \\ College of Engineering Poonjar \\ Kottyam, Kerala - India
}

\begin{abstract}
Automatic Image annotation is a dominant research area in computer science. It is concerned with the storage of images and assigning meaningful keywords to it. There are several methods developed for efficient automatic image annotation which uses various optimization techniques. The purpose of this paper is to show the survey study done on the optimization techniques for Image annotation. An image has several preeminent characteristics like colour texture, shape etc. These different descriptors of the images can form a combined feature vector. Optimization algorithms such as Particle swam optimization algorithm, Genetic algorithm etc can be used for optimum feature selection.
\end{abstract}

\section{General Terms}

Automatic Image Annotation, Image retrieval, Optimization

\section{Keywords}

Image Annotation, Optimization, Particle Swam Optimization, Content based image retrieval, Feature Extraction

\section{INTRODUCTION}

With the advent of various multimedia devices such as digital cameras, mobile phones etc.. The number of images has increased dramatically. Thus, Effective methods are required for organizing, searching and browsing these images. Many search engines use text-based searching methods for retrieving images. Indexing images based on its semantic content will improve the image search quality. However, as it is impossible to manually annotate all images, Automatic image annotation (AIA) might be a promising solution. The goal of Automatic image annotation is to assign meaningful keywords to an image by automatically checking the semantics of the image. Automatic image annotation is essential to label a huge collection of unlabeled photos. Traditional methods of image annotation are not adequate as the amount of images to be indexed is huge, which makes it impractical and error prone. Hence, it has become an urgent need for developing novel and effective paradigms that go beyond these conventional approaches. Thus to enhance annotation performance, optimization techniques are used. Optimization is a commonly encountered mathematical problem in all engineering disciplines. It means finding the best possible solution. Feature weighting is a technique used to approximate the optimal degree of influence of individual features. In this survey various methods to annotate images are discussed. Also, various approaches for optimum feature selection are interpreted and their limitations are discussed.

\section{FEATURE DESCRIPTORS FOR IMAGE ANNOTATION/RETRIEVAL}

A feature is a significant piece of information extracted from an image which is used to interpret an image. Image feature extraction is a type of dimensionality reduction that detect and isolate various desired portions of a digitized image. An image has several visual attributes such as color, texture, shape etc.

Color is one of low-level visual features, which is commonly used for image processing. It is invariant in image scaling, rotation and translation and has the characteristics of easy calculation. The comparison of color histograms[3][5][6] is one of the most widely used techniques for image annotation. A histogram is the distribution of the number of pixels of an image. The number of elements in a histogram relates to the number of bits in each pixel of an image. RGB[4] color model (RGB: Red, Green, Blue) is the most commonly used color model. It is an additive color model: the colors red, green, and blue are combined to generate other colors. It is not perceptually uniform this means that the measure of the variation perceived by a human is different from the mathematical distance. HSV (HSL, HSB)[1] models are much closer to human eye perception of color The components of these models are: Hue, Saturation, and Value (lightness or brightness). The hue represents the chromatic component in this model. Saturation refers to the predominance of a particular hue in a color. The value of a color refers to the intensity (the lightness or the darkness of the color). Color moments[6] are measures that can be used distinguish images based on their features of color. It represents the mean, variance and standard deviation of an image.

Texture can be a very useful feature For browsing, searching and retrieval of images,Texture descriptor provides measures of the properties such as smoothness, coarseness, and regularity etc. Texture is usually Denoted by the values of energy, entropy, contrast and homogeneity. gray-level cooccurrence matrix (GLCM)[11] examines texture based on the spatial relationship of pixels. Discrete Wavelet Transform (DWT)[3][6][9] are used for analysis of textures recorded With different resolution. Discrete Cosine Transform (DCT) [7][9] is a powerful transform to extract features from face images.

Histogram of Oriented Gradient (HOG)[1] descriptor technique counts occurrences of gradient orientation in localized portions of an image - detection window, or region of interest. SIFT[10] has been proven to be the most robust local invariant feature descriptor. It is designed mainly for gray images. However, color provides valuable information in object description and matching tasks. 
Shape descriptor is a set of numbers that are used to describe a given shape. Shape aspect can be achieved by using gradient operators or morphological operators. Shape features can be obtained by gradient vector flow (GVF), chain code, edge histogram descriptor (EHD), and Zernike moments, contour co-occurance matrix (CCM)[4] and edge co-occurance Matrix $(\mathrm{ECM})$ are some histogram based shape descriptors.

\section{CLASSIFICATION METHODS}

Image classification analyzes the numerical properties of various image features and organizes data into categories. Classification algorithms typically employ two phases of processing: training and testing. In the initial training phase, characteristic properties of typical image features are isolated and, based on these, a unique description of each classification category, i.e. training class, is created. In the subsequent testing phase, these feature-space partitions are used to classify image features.

\subsection{Support vector machine (SVM)}

A Support Vector Machine (SVM)[4] is a discriminative classifier formally defined by a separating hyper plane. It is a vector space based machine learning method where the goal is to find a decision boundary between two classes that is maximally far from any point in the training data. Optimization techniques can be used along with SVM classifiers to enhance the retrieval performance.

\subsection{Minimum distance classifier}

The minimum distance classifier[10] is used to classify unknown image data into classes which minimize the distance between the image data and the class in multi-feature space. The distance is defined as an index of similarity so that the minimum distance is identical to the maximum similarity. Euclidian distance, Normalized Euclidian distance, Mahalanobis distance, Manhattan distance etc are some of the distance measurements that are often used by minimum distance classifiers.

\subsection{K-nearest neighbour classifier}

k-Nearest Neighbors[4][11] is a classification method that stores all available cases and classifies new cases based on a similarity measure (e.g., distance functions). Euclidian distance, Manhattan distance, minkowski distance are some of the distance measurements used. The input consists of the $\mathrm{k}$ closest training examples in the feature space and the output is a class membership. An object is classified by a majority vote of its neighbours, with the object being assigned to the class most common among its $\mathrm{k}$ nearest neighbours.

\section{OPTIMIZATION}

Optimization is a frequently encountered mathematical problem in all engineering disciplines. It means finding the best possible solution. Optimization of image annotation techniques are an important research area in computer science. Optimization can be achieved in different phases of annotation such as feature extraction, feature selection and weighted feature selection. In feature extraction phase optimum features are extracted from an image using an optimization algorithm along with feature extraction techniques. In [4] feature extraction process is done using Particle swam optimization with SVM classifier. In feature selection, optimization algorithms such as particle swam optimization, genetic algorithm, Firefly algorithm etc are employed. In [9] and [10] a PSO-based feature selection algorithm is utilized to search the feature space for the optimal feature subset. Instead of assigning equal weights to different features, weights are appropriately assigned using optimization algorithms such as Genetic algorithm, Particle Swam Optimization etc which gives an optimized feature vector of each image.

\section{STATE OF THE ART IN OPTIMIZATION FOR IMAGE ANNOTATION}

Sreekumar. K and Ajimi Ameer[1] proposed a method for Efficient Automatic Image Annotation using Optimized weighted Complementary Feature Fusion using Genetic Algorithm. First, SURF, HSV and HOG features are obtained from the training set images and an average feature descriptor representing each class is obtained. Feature vectors are clustered into $\mathrm{k}$ clusters using $\mathrm{K}$ means clustering. Instead of assigning equal weights to different features, weights are appropriately assigned using Genetic algorithm which gives an optimized feature vector of each image. As the number of iterations increases in genetic algorithm, weights obtained becomes more accurate and yields better result.

Dong Yang and Ping Guo[2] focused on Image modelling with combined optimization techniques for image semantic annotation. In this framework, low-level image features are extracted from sub-blocks of a given image. Affinity propagation algorithm is applied to estimate the image feature distribution. Then a Bayesian classifier is built using Gaussian mixture model for image semantic annotation. A training data optimization algorithm is developed to improve the accuracy of the Bayesian classifier.

Darsana B and G. Jagajothi[3] proposed a novel approach of, distributed retrieval of images using particle swarm optimization and Hadoop file systems. It deals with problems of semantic gap and delayed response time in content based image retrieval by coalescing automatic relevance feedback, a stochastic algorithm and distribution of image retrieval. first the similarity evaluation of training image features and query image features are done. Then a combined similarity evaluation is done on the retrieved images to separate relevant and non relevant images. Particle swam optimization is used to assign weights to features.

Vinay Kumar Lowanshi, Shweta Shrivastava and Vineet Richhariya[4] proposed novel approach for content based image retrieval. It works in two phases. In the first phase feature extraction process is done using Particle swam optimization with SVM classifier, after successful classification in first phase the retrieved results are passed into the second phase classifier. And in the second phase $\mathrm{KNN}$ classifier is used and Genetic Algorithm is applied with it to produce a more accurate result. and it state that the accuracy of proposed approach is $97.33 \%$ and existing is $83.33 \%$ on the same dataset.

Lei Wang and Latifur Khan[5] proposed a method for Automatic Image Annotation and Retrieval Using Weighted Feature Selection. For a given cluster, relevant features determined based on histogram analysis and assign greater weight to relevant features as compared to less relevant features. Lei Wang and Latifur Khan implemented various different models to link visual tokens with keywords based on the clustering results of K-means algorithm with weighted feature selection and without feature selection.

An adaptive content based image retrieval (CBIR) approach based on relevance feedback and Firefly algorithm is 
proposed by T.Kanimozhi and K.Latha[6]. In addition to the color descriptor, wavelet-based texture descriptor is considered to improve the retrieval performance. It is a relevance feedback (RF) based approach and optimization is done using firefly algorithm. firefly has a deeper precision accuracy than GA based optimization

C.Ramesh babu durai, V.Duraisamy and C.Vinothkumar[7] focused on Improved Content Based Image Retrieval Using Neural Network Optimization with Genetic Algorithm. Here feature extraction is done using Discrete cosine Transform. Gaussian Fuzzy Feed Forward Neural Network algorithm is used for classification and optimize the momentum and learning rate using Genetic algorithm. The classification accuracy obtained is $96.29 \%$.

S. Bahrami and M. Saniee Abadeh[8] tried to propose an automated annotation based method to solve image annotation problem. Here Genetic algorithm is utilized for feature selection ,Multi-Label KNN algorithm to weight neighbours and to generate a novel weighted matrix.

Rabab M. Ramadan and Rehab F. Abdel - Kader[9] proposed a method for Face Recognition Using Particle Swarm Optimization-Based Selected Features. The proposed PSObased feature selection algorithm is utilized to search the feature space for the optimal feature subset. The algorithm is applied to coefficients extracted by two feature extraction techniques: the discrete cosine transforms (DCT) and the discrete wavelet transform (DWT). The performance of the proposed algorithm is compared to the performance of a GAbased feature selection algorithm and was found to yield better results with less number of selected features.
Ayan Seal et.al.[10] Introduced a robust method for face recognition using thermal face images. In the first step, thermal human face image is pre-processed and facial region from the entire image is cropped. In the next step, scale invariant feature transform (SIFT) is used to extract the features from the cropped face region. a PSO based feature selection algorithm is efficiently utilized to search the optimum features.

S. Sivakumar and Dr.C.Chandrasekar[11] proposed a continuous particle swarm optimization (PSO) to implement a feature selection in wrapper based method, and the k-nearest neighbour classification serve as a fitness function of PSO for the classification problem. It increases the classification accuracy and, at the same time, keeps computational resources needed to a minimum.

Bae-Muu Chang, Hung-Hsu Tsai and Wen-Ling Chou presents a content-based image retrieval method using three kinds of visual features and 12 distance measurements, which is optimized by particle swarm optimization (PSO) algorithm. Firstly, three kinds of features such as color, texture, and shape are extracted from the image. To calculate similarities between query image and images in the database, appropriate distance measurements for each kind of features are used. PSO algorithm is utilized for finding the approximately optimal weights for three similarities with respect to three kinds of features.

TABLE 1 Comparison of different optimization techniques

\begin{tabular}{|c|c|c|c|c|c|c|}
\hline Author & $\begin{array}{l}\text { Public } \\
\text { ation } \\
\text { Year }\end{array}$ & $\begin{array}{c}\text { Feature } \\
\text { extraction } \\
\text { method }\end{array}$ & $\begin{array}{c}\text { Optimization } \\
\text { method }\end{array}$ & $\begin{array}{l}\text { Classificatio } \\
\text { n method }\end{array}$ & Database & Advantages/Disadvantages \\
\hline $\begin{array}{l}\text { SreeKumar.K, } \\
\text { Ajimi Ameer }\end{array}$ & 2015 & SURF,HOG, HSV & $\begin{array}{l}\text { Genetic } \\
\text { Algorithm }\end{array}$ & $\begin{array}{l}\text { K-Means } \\
\text { Clustering }\end{array}$ & $\begin{array}{l}\text { Corel } 1000 \\
\text { dataset }\end{array}$ & $\begin{array}{l}\text { As the number of iterations } \\
\text { increases in genetic } \\
\text { algorithm, weights obtained } \\
\text { becomes much more accurate } \\
\text { and yields better result. }\end{array}$ \\
\hline $\begin{array}{l}\text { Lei Wang, Latifur } \\
\text { Khan }\end{array}$ & 2004 & $\begin{array}{l}\text { Histogram } \\
\text { analysis }\end{array}$ & $\begin{array}{l}\text { Weighted } \\
\text { feature } \\
\text { selection }\end{array}$ & $\begin{array}{l}\text { K-Means } \\
\text { Clustering }\end{array}$ & Duygulu02 & $\begin{array}{l}\text { Better annotation performance } \\
\text { and correspondence accuracy }\end{array}$ \\
\hline $\begin{array}{l}\text { Darsana B } \\
\text { G. Jagajothi }\end{array}$ & 2013 & $\begin{array}{l}\text { Color histogram } \\
\text { bins } \\
\text { wavelet texture } \\
\text { energy values. }\end{array}$ & $\begin{array}{l}\text { Particle swam } \\
\text { optimization }\end{array}$ & $\begin{array}{l}\text { Similarity } \\
\text { evaluation }\end{array}$ & $\begin{array}{l}\text { Corel image } \\
\text { database, } \\
\text { SIMPLicity, } \\
\text { web images. }\end{array}$ & $\begin{array}{l}\text { Dynamically modifies the } \\
\text { feature space by feeding } \\
\text { automatic } \\
\text { relevance feedback and } \\
\text { clustering relevant images } \\
\text { using metaheuristics. }\end{array}$ \\
\hline $\begin{array}{l}\text { Vinay Kumar } \\
\text { Lowanshi, } \\
\text { Shweta }\end{array}$ & 2014 & $\begin{array}{l}\text { RGB histogram } \\
\text { CCM/CCH }\end{array}$ & $\begin{array}{l}\text { Particle swam } \\
\text { optimization }\end{array}$ & $\begin{array}{l}\text { SVM and } \\
\text { kNN }\end{array}$ & $\begin{array}{l}\text { Corel } 1000 \\
\text { dataset }\end{array}$ & $\begin{array}{l}\text { Improved accuracy than } \\
\text { existing approach }\end{array}$ \\
\hline
\end{tabular}




\begin{tabular}{|c|c|c|c|c|c|c|}
\hline $\begin{array}{l}\text { Shrivastava } \\
\text { Vineet } \\
\text { Richhariya }\end{array}$ & & & & classifier & & \\
\hline $\begin{array}{l}\text { T.Kanimozhi, } \\
\text { K.Latha }\end{array}$ & 2013 & $\begin{array}{l}\text { Color histogram, } \\
\text { Color moment, } \\
\text { edge direction } \\
\text { histogram, } \\
\text { wavelet texture } \\
\text { feature }\end{array}$ & $\begin{array}{l}\text { Firefly } \\
\text { algorithm }\end{array}$ & $\begin{array}{l}\text { Euclidian } \\
\text { distance }\end{array}$ & Corel database & $\begin{array}{l}\text { Highly efficient, robust and } \\
\text { highly rapid for image } \\
\text { accuracy } \\
\text { based application. }\end{array}$ \\
\hline $\begin{array}{l}\text { C.Ramesh babu } \\
\text { durai, } \\
\text { V.Duraisamy, } \\
\text { C.Vinothkumar }\end{array}$ & 2012 & $\begin{array}{l}\text { Discrete Cosine } \\
\text { Transform }\end{array}$ & $\begin{array}{l}\text { Genetic } \\
\text { algorithm }\end{array}$ & $\begin{array}{l}\text { Fuzzy Feed } \\
\text { Forward } \\
\text { Neural } \\
\text { Network }\end{array}$ & $\begin{array}{l}\text { MRI medical } \\
\text { image }\end{array}$ & Efficient, robust \\
\hline $\begin{array}{l}\text { Rabab M. } \\
\text { Ramadan, Rehab } \\
\text { F. Abdel - Kader }\end{array}$ & 2009 & $\begin{array}{l}\text { Discrete cosine } \\
\text { transforms, } \\
\text { Discrete wavelet } \\
\text { transform }\end{array}$ & $\begin{array}{l}\text { Particle swam } \\
\text { optimization }\end{array}$ & $\begin{array}{l}\text { Euclidean } \\
\text { distance }\end{array}$ & Face images & $\begin{array}{l}\text { Generating excellent } \\
\text { recognition } \\
\text { accuracy with the minimal set } \\
\text { of selected features. }\end{array}$ \\
\hline Ayan Seal, et.al & 2015 & SIFT & $\begin{array}{l}\text { Particle swam } \\
\text { optimization }\end{array}$ & $\begin{array}{l}\text { Minimum } \\
\text { distance } \\
\text { classifier }\end{array}$ & $\begin{array}{l}\text { UGC-JU } \\
\text { thermal face } \\
\text { database }\end{array}$ & $\begin{array}{l}\text { PSO based } \\
\text { feature selection algorithm is } \\
\text { efficiently utilized to search } \\
\text { the optimum } \\
\text { features which are not noisy } \\
\text { and also not irrelevant }\end{array}$ \\
\hline $\begin{array}{l}\text { S. Sivakumar, } \\
\text { Dr.C.Chandrasek } \\
\text { ar }\end{array}$ & 2014 & $\begin{array}{l}\text { GLCM } \\
\text { GLRLM }\end{array}$ & $\begin{array}{l}\text { Particle swam } \\
\text { optimization }\end{array}$ & $\begin{array}{l}\mathrm{k}-\mathrm{NN} \\
\text { classifier }\end{array}$ & $\begin{array}{l}\text { LIDC-IDRI } \\
\text { Lung CT scan } \\
\text { images }\end{array}$ & $\begin{array}{l}\text { It increases the } \\
\text { classification accuracy and, at } \\
\text { the same time, keeps } \\
\text { computational resources } \\
\text { needed to a minimum. }\end{array}$ \\
\hline
\end{tabular}

\section{CONCLUSION}

This paper, attempts to provide a comprehensive survey on the latest developments of Automatic Image Annotation techniques with a special emphasis on Optimization. Optimization can be achieved in different phases of annotation such as feature extraction, feature selection and weighted feature selection. From this survey a method for efficient Automatic image annotation is proposed. An image has several dominant characteristics like color texture, shape etc. These different descriptors for the images can form a combined feature vector. However, in order to have optimum performance particle swam optimization algorithm (PSO) based feature selection may be used.

\section{REFERENCES}

[1] Ajimi Ameer and SreeKumar.K, Efficient Automatic Image Annotation using Optimized weighted Complementary Feature Fusion using Genetic Algorithm, Second International Symposium on Computer Vision and the Internet (VisionNet'15)

[2] Dong Yang and Ping Guo, Image modeling with combined optimization techniques for image semantic annotation, Springer-Verlag London Limited 2010
[3] Darsana B and G. Jagajothi, Distributed Retrieval of Images using Particle Swarm Optimization and Hadoop, international Journal of Computer Applications (0975 8887) Volume 71-No.8, May 2013

[4] Vinay Kumar Lowanshi, Shweta Shrivastava and Vineet Richhariya, An Efficient Approach for Content based Image Retrieval using SVM, KNN-GA as Multilayer Classifier, International Journal of Computer Applications (0975 - 8887) Volume 107 - No. 21, December 2014

[5] Lei Wang and Latifur Khan, Automatic Image Annotation and Retrieval Using Weighted Feature Selection , Multimedia Software Engineering, 2004. Proceedings. IEEE Sixth International Symposium

[6] T.Kanimozhi and K.Latha, A Meta-Heuristic Optimization Approach for Content Based Image Retrieval using Relevance Feedback Method, Proceedings of the World Congress on Engineering 2013 Vol II, WCE 2013, July 3 - 5, 2013, London, U.K.

[7] C.Ramesh babu durai, V.Duraisamy and C.Vinothkumar, Improved Content Based Image 
Retrieval Using Neural Network Optimization with Genetic Algorithm, International Journal of Emerging Technology and Advanced Engineering (ISSN 22502459, Volume 2, Issue 7, July 2012)

[8] S. Bahrami and M. Saniee Abadeh, Automatic image annotation using an evolutionary algorithm, Telecommunications (IST), $2014 \quad 7$ th International Symposium

[9] Rabab M. Ramadan and Rehab F. Abdel - Kader, Face Recognition Using Particle Swarm Optimization-Based Selected Features, International Journal of Signal Processing, Image Processing and Pattern Recognition Vol. 2, No. 2, June 2009

[10] Ayan Seal, Suranjan Ganguly, Debotosh Bhattacharjee, Mita Nasipuri and Consuelo Gonzalo-Martin, Feature
Selection using Particle Swarm Optimization for Thermal Face Recognition, Springer India 2015 R. Chaki et al. (eds.), Applied Computation and Security Systems, Advances in Intelligent Systems and Computing 304, DOI 10.1007/978-81-322-1985-9_2

[11] Sivakumar and Dr.C.Chandrasekar, Modified PSO Based Feature Selection for Classification of Lung CT Images, International Journal of Computer Science and Information Technologies, Vol. 5 (2) , 2014, 2095-2098

[12] Bae-Muu Chang,Hung-Hsu Tsai and Wen-Ling Chou, Using visual features to design a content-based image retrieval method optimized by particle swarm optimization algorithm 